International Journal of Modern Physics C,

(C) World Scientific Publishing Company

\title{
REALISTIC MODELING OF STRONGLY CORRELATED ELECTRON SYSTEMS: AN INTRODUCTION TO THE LDA+DMFT APPROACH
}

\author{
K. Held ${ }^{1,3}$, I.A. Nekrasov ${ }^{2}$, N. Blümer ${ }^{1}$, V.I. Anisimov ${ }^{2}$, and D. Vollhardt ${ }^{1}$ \\ ${ }^{1}$ Theoretical Physics III, Center for Electronic Correlations and Magnetism \\ Institute for Physics, University of Augsburg, D-86135 Augsburg, Germany \\ ${ }^{2}$ Institute of Metal Physics, Russian Academy of Sciences-Ural Division \\ 620219 Yekaterinburg GSP-170, Russia \\ ${ }^{3}$ Present address: Physics Department, Princeton University \\ Princeton, NJ 08544, USA
}

Received (received date)

\begin{abstract}
The LDA+DMFT approach merges conventional band structure theory in the local density approximation (LDA) with a state-of-the-art many-body technique, the dynamical mean-field theory (DMFT). This new computational scheme has recently become a powerful tool for $a b$ initio investigations of real materials with strong electronic correlations. In this paper an introduction to the basic ideas and the set-up of the LDA+DMFT approach is given. Results for the photoemission spectra of the transition metal oxide $\mathrm{La}_{1-x} \mathrm{Sr}_{x} \mathrm{TiO}_{3}$, obtained by solving the DMFT-equations by quantum Monte-Carlo (QMC) simulations, are presented and are found to be in very good agreement with experiment. The numerically exact $\mathrm{DMFT}(\mathrm{QMC})$ solution is compared with results obtained by two approximative solutions, i.e., the iterative perturbation theory and the non-crossing approximation.
\end{abstract}

Keywords: 71.27.+a Strongly correlated electron systems; heavy fermions; 74.25.Jb Electronic structure; 79.60.-i Photoemission and photoelectron spectra

\section{Introduction}

It is an established fact of solid state physics that many electronic properties of matter are well described by the purely electronic Hamiltonian

$$
\begin{aligned}
\hat{H}= & \sum_{\sigma} \int d^{3} r \hat{\Psi}^{+}(\mathbf{r}, \sigma)\left[-\frac{\hbar^{2}}{2 m_{e}} \Delta+V_{\mathrm{ion}}(\mathbf{r})\right] \hat{\Psi}(\mathbf{r}, \sigma) \\
& +\frac{1}{2} \sum_{\sigma \sigma^{\prime}} \int d^{3} r d^{3} r^{\prime} \hat{\Psi}^{+}(\mathbf{r}, \sigma) \hat{\Psi}^{+}\left(\mathbf{r}^{\prime}, \sigma^{\prime}\right) V_{\mathrm{ee}}\left(\mathbf{r}-\mathbf{r}^{\prime}\right) \hat{\Psi}\left(\mathbf{r}^{\prime}, \sigma^{\prime}\right) \hat{\Psi}(\mathbf{r}, \sigma),
\end{aligned}
$$

where the crystal lattice enters only through an ionic potential. The applicability of this approach may be justified by the validity of the Born and Oppenheimer approximationl. Here, $\hat{\Psi}^{+}(\mathbf{r}, \sigma)$ and $\hat{\Psi}(\mathbf{r}, \sigma)$ are field operators that create and 
annihilate an electron at position $\mathbf{r}$ with spin $\sigma, \Delta$ is the Laplace operator, $m_{e}$ the electron mass, $e$ the electron charge, and

$$
V_{\mathrm{ion}}(\mathrm{r})=-e^{2} \sum_{i} \frac{Z_{i}}{\left|\mathbf{r}-\mathbf{R}_{\mathbf{i}}\right|} \quad \text { and } \quad V_{\mathrm{ee}}\left(\mathbf{r}-\mathbf{r}^{\prime}\right)=\frac{e^{2}}{2} \sum_{\mathbf{r} \neq \mathbf{r}^{\prime}} \frac{1}{\left|\mathbf{r}-\mathbf{r}^{\prime}\right|}
$$

denote the one-particle potential due to all ions $i$ with charge $e Z_{i}$ at given positions $\mathbf{R}_{\mathbf{i}}$, and the electron-electron interaction, respectively.

While the $a b$ initio Hamiltonian (1) is easy to write down it is impossible to solve, even numerically, if more than a few electrons are involved. The reason for this is the electron-electron interaction which correlates every electron with all others. Therefore, one either needs to make substantial approximations to deal with the Hamiltonian (11), or replace it by a strongly simplified model Hamiltonian. At present these different strategies for the investigation of the electronic properties of solids are applied by two largely separate groups: the density functional theory (DFT) and the many-body community. It is known for a long time already that DFT, together with its local density approximation (LDA), is a highly successful technique for the calculation of the electronic structure of many real materials 2 . However, for strongly correlated materials, i.e., $d$ - and $f$-electron system which have a Coulomb interaction comparable to the band-width, DFT/LDA is seriously restricted in its accuracy and reliability. Here, the model Hamiltonian approach is more general and powerful since there exist systematic theoretical techniques to investigate the many-electron problem with increasing accuracy. These manybody techniques allow to describe qualitative tendencies and understand the basic mechanism of various physical phenomena. At the same time the model Hamiltonian approach is seriously restricted in its ability to make quantitative predictions since the input parameters are not accurately known and hence need to be adjusted. One of the most successful techniques in this respect is the dynamical mean-field theory (DMFT) - a non-perturbative approach to strongly correlated electron systems which was developed during the past decade 4.6 . The LDA+DMFT approach, which was first formulated by Anisimov et al. 1 , combines the strength of DFT/LDA to describe the weakly correlated part of the ab initio Hamiltonian (11), i.e., electrons in $s$ - and $p$-orbitals as well as the long-range interaction of the $d$ - and $f$-electrons, with the power of DMFT to describe the strong correlations induced by the local Coulomb interaction of the $d$ - or $f$-electrons.

Starting from the $a b$ initio Hamiltonian (1), the LDA+DMFT approach is derived in section 2. As a particular example, the LDA+DMFT calculation for $\mathrm{La}_{1-x} \mathrm{Sr}_{x} \mathrm{TiO}_{3}$ is discussed in section 3. Furthermore, the LDA+DMFT spectrum is calculated by means of numerically exact quantum Monte Carlo (QMC) simulations, and is compared to that obtained within two approximations commonly employed to solve the DMFT, i.e., the iterated perturbation theory (IPT) and the non-crossing approximation (NCA). A discussion of the LDA+DMFT approach and its future prospects (section 4) closes the presentation. 


\section{The LDA+DMFT approach}

\subsection{Density functional theory}

The fundamental theorem of DFT by Hohenberg and Kohn 8 states that the ground state energy is a functional of the electron density which assumes its minimum at the ground state electron density. Following Levy and the functional even constructed by taking the minimum (infimum) of the energy expectation value w.r.t. all (many-body) wave functions $\varphi\left(\mathbf{r}_{1} \sigma_{1}, \ldots \mathbf{r}_{\mathbf{N}} \sigma_{N}\right)$ at a given electron number $N$ which yield the electron density $\rho(\mathbf{r})$ :

$$
E[\rho]=\inf \left\{\langle\varphi|\hat{H}| \varphi\rangle \mid\left\langle\varphi\left|\sum_{i=1}^{N} \delta\left(\mathbf{r}-\mathbf{r}_{\mathbf{i}}\right)\right| \varphi\right\rangle=\rho(\mathbf{r})\right\}
$$

However, this construction is of no practical value since it actually requires the evaluation of the Hamiltonian (11). Only certain contributions like the Hartree energy $E_{\text {Hartree }}[\rho]=\frac{1}{2} \int d^{3} r^{\prime} d^{3} r V_{\mathrm{ee}}\left(\mathbf{r}-\mathbf{r}^{\prime}\right) \rho\left(\mathbf{r}^{\prime}\right) \rho(\mathbf{r})$ and the energy of the ionic potential $E_{\text {ion }}[\rho]=\int d^{3} r V_{\text {ion }}(\mathbf{r}) \rho(\mathbf{r})$ can be expressed directly in terms of the electron density. This leads to

$$
E[\rho]=E_{\text {kin }}[\rho]+E_{\text {ion }}[\rho]+E_{\text {Hartree }}[\rho]+E_{\text {xc }}[\rho],
$$

where $E_{\text {kin }}[\rho]$ denotes the kinetic energy, and $E_{\mathrm{xc}}[\rho]$ is the unknown exchange and correlation term which contains the energy of the electron-electron interaction except for the Hartree term. Hence all the difficulties of the many-body problem have been transfered into $E_{\mathrm{xc}}[\rho]$. While the kinetic energy $E_{\text {kin }}$ cannot be expressed explicitly in terms of the electron density one can employ a trick to determine it. Instead of minimizing $E[\rho]$ w.r.t. $\rho$ one minimizes it w.r.t. a set of one-particle wave functions $\varphi_{i}$ related to $\rho$ via

$$
\rho(\mathbf{r})=\sum_{i=1}^{N}\left|\varphi_{i}(\mathbf{r})\right|^{2}
$$

To guarantee the normalization of $\varphi_{i}$, the Lagrange parameters $\varepsilon_{i}$ are introduced such that the variation $\delta\left\{E[\rho]+\varepsilon_{i}\left[1-\int d^{3} r\left|\varphi_{i}(\mathbf{r})\right|^{2}\right]\right\} / \delta \varphi_{i}(\mathbf{r})=0$ yields the KohnSham 10 equations:

$$
\left[-\frac{\hbar^{2}}{2 m_{e}} \Delta+V_{\mathrm{ion}}(\mathbf{r})+\int d^{3} r^{\prime} \rho\left(\mathbf{r}^{\prime}\right) V_{\mathrm{ee}}\left(\mathbf{r}-\mathbf{r}^{\prime}\right)+\frac{\delta E_{\mathrm{xc}}[\rho]}{\delta \rho(\mathbf{r})}\right] \varphi_{i}(\mathbf{r})=\varepsilon_{i} \varphi_{i}(\mathbf{r}) .
$$

These equations have the same form as a one-particle Schrödinger equation which, a posteriori, justifies to calculate the kinetic energy by means of the one-particle wave-function ansatz. The kinetic energy at the ground state density is, then, given by $E_{\text {kin }}\left[\rho_{\text {min }}\right]=-\sum_{i=1}^{N}\left\langle\varphi_{i}\left|\hbar^{2} \Delta /\left(2 m_{e}\right)\right| \varphi_{i}\right\rangle$ if the $\varphi_{i}$ are the self-consistent (spindegenerate) solutions of Eqs. (6) and (5) with lowest "energy" $\epsilon_{i}$. Note, however, 
that the one-particle potential of Eq. (6), i.e.,

$$
V_{\mathrm{ion}}(\mathbf{r})+\int d^{3} r^{\prime} \rho\left(\mathbf{r}^{\prime}\right) V_{\mathrm{ee}}\left(\mathbf{r}-\mathbf{r}^{\prime}\right)+\frac{\delta E_{\mathrm{xc}}[\rho]}{\delta \rho(\mathbf{r})},
$$

is only an auxiliary potential which artificially arises in the approach to minimize $E[\rho]$. Thus, the wave functions $\varphi_{i}$ and the Lagrange parameters $\varepsilon_{i}$ have no physical meaning at this point.

\subsection{Local density approximation}

So far no approximations have been employed since the difficulty of the many-body problem was only transferred to the unknown functional $E_{x c}[\rho]$. For this term the local-density approximation (LDA) which approximates the functional $E_{x c}[\rho]$ by a function that depends on the local density only, i.e.,

$$
E_{x c}[\rho] \rightarrow \int d^{3} r E_{x c}^{\mathrm{LDA}}(\rho(\mathbf{r}))
$$

was found to be unexpectedly successful. Here, $E_{x c}^{\mathrm{LDA}}(\rho(\mathbf{r}))$ is usually calculated from the Hartree-Fock solution or the numerical simulation of the jellium problem which is defined by $V_{\text {ion }}(\mathbf{r})=$ const 15 .

In principle DFT/LDA only allows one to calculate static properties like the ground state energy or its derivatives. However, one of the major applications of LDA is the calculation of band structures. To this end, the Lagrange parameters $\varepsilon_{i}$ are interpreted as the physical (one-particle) energies of the system under consideration. Since the true ground-state is not a simple one-particle wave-function, this is a further approximation beyond DFT. Actually, this approximation corresponds to the replacement of the Hamiltonian (1) by

$$
\begin{aligned}
\hat{H}_{\mathrm{LDA}}=\sum_{\sigma} \int d^{3} r \hat{\Psi}^{+}(\mathbf{r}, \sigma)[ & -\frac{\hbar^{2}}{2 m_{e}} \Delta+V_{\mathrm{ion}}(\mathbf{r})+\int d^{3} r^{\prime} \rho\left(\mathbf{r}^{\prime}\right) V_{\mathrm{ee}}\left(\mathbf{r}-\mathbf{r}^{\prime}\right) \\
& \left.+\frac{\delta E_{x c}^{\mathrm{LDA}}[\rho]}{\delta \rho(\mathbf{r})}\right] \hat{\Psi}(\mathbf{r}, \sigma)
\end{aligned}
$$

For practical calculations one needs to expand the field operators w.r.t. a basis $\Phi_{i l m}$, e.g., a linearized muffin-tin orbital (LMTO) 12 basis (here $i$ denotes lattice sites; $l$ and $m$ are orbital indices). In this basis,

$$
\hat{\Psi}^{+}(\mathbf{r}, \sigma)=\sum_{i l m} \hat{c}_{i l m}^{\sigma \dagger} \Phi_{i l m}(\mathbf{r})
$$

such that the Hamiltonian (9) reads

$$
\hat{H}_{\mathrm{LDA}}=\sum_{i l m, j l^{\prime} m^{\prime}, \sigma}\left(\delta_{i l m, j l^{\prime} m^{\prime}} \varepsilon_{i l m} \hat{n}_{i l m}^{\sigma}+t_{i l m, j l^{\prime} m^{\prime}} \hat{c}_{i l m}^{\sigma \dagger} \hat{c}_{j l^{\prime} m^{\prime}}^{\sigma}\right) .
$$


Here, $\hat{n}_{i l m}^{\sigma}=\hat{c}_{i l m}^{\sigma \dagger} \hat{c}_{i l m}^{\sigma}$

$$
t_{i l m, j l^{\prime} m^{\prime}}=\left\langle\Phi_{i l m}\left|-\frac{\hbar^{2} \Delta}{2 m_{e}}+V_{\mathrm{ion}}(\mathbf{r})+\int d r^{\prime} \rho\left(\mathbf{r}^{\prime}\right) V_{\mathrm{ee}}\left(\mathbf{r}-\mathbf{r}^{\prime}\right)+\frac{\delta E_{x c}^{\mathrm{LDA}}[\rho]}{\delta \rho(\mathbf{r})}\right| \Phi_{j l^{\prime} m^{\prime}}\right\rangle
$$

for $i l m \neq j l^{\prime} m^{\prime}$ and zero otherwise; $\varepsilon_{i l m}$ denotes the corresponding diagonal part.

As for static properties LDA band structure calculations are also often highly successful - but only for weakly correlated materials 2 . Indeed, the self-consistent solution of the one-particle Hamiltonian $\hat{H}_{\mathrm{LDA}}$ (11) together with Eq. (5) treats electronic correlations only rudimentarily. Consequently, LDA is not reliable when applied to correlated materials, and can even be completely wrong. For example, it predicts the antiferromagnetic insulator $\mathrm{La}_{2} \mathrm{CuO}_{4}$ to be a non-magnetic metal 3 .

\subsection{Supplementing LDA with local Coulomb correlations}

Of prime importance for correlated materials are the local Coulomb interactions between $d$ - and $f$-electrons on the same lattice site since these contributions are largest. This is due to the extensive overlap of these localized orbitals which results in strong correlations 14 . To correct for these contributions, one can supplement the LDA Hamiltonian (11) with the local Coulomb interaction $U_{m m^{\prime}}^{\sigma \sigma^{\prime}}$ between the localized electrons (for which we assume $i=i_{d}$ and $l=l_{d}$ ):

$$
\hat{H}_{\mathrm{LDA}+\mathrm{correl}}=\hat{H}_{\mathrm{LDA}}+\frac{1}{2} \sum_{i=i_{d}, l=l_{d}, m \sigma m^{\prime} \sigma}^{\prime} U_{m m^{\prime}}^{\sigma \sigma^{\prime}} \hat{n}_{i l m \sigma} \hat{n}_{i l m^{\prime} \sigma^{\prime}}-\hat{H}_{\mathrm{LDA}}^{U}
$$

Here, the prime on the sum indicates that at least two of the indices of an operator have to be different, and a term $\hat{H}_{\mathrm{LDA}}^{U}$ is substracted to avoid double-counting of those contributions of the local Coulomb interaction already contained in $\hat{H}_{\mathrm{LDA}}$. Since there does not exist a direct microscopic or diagrammatic link between the model Hamiltonian approach and LDA it is not possible to express $\hat{H}_{\mathrm{LDA}}^{U}$ rigorously in terms of $U$ and $\rho$. Guided by the observation that the LDA calculates the total energy of isolated atoms rather well, it was argued 11 that the average energy $E_{\mathrm{LDA}}^{U}$ corresponding to $\hat{H}_{\mathrm{LDA}}^{U}$ is well approximated by the energy of the interaction term in the atomic limit. Hence, in the case of an orbital- and spin-independent $U_{m m^{\prime}}^{\sigma \sigma^{\prime}}=U$ one may write

$$
E_{\mathrm{LDA}}^{U}=\frac{1}{2} U n_{d}\left(n_{d}-1\right) .
$$

(For the corresponding equation including Hund's rule coupling see Ref. Q). Here, $n_{d}=\sum_{m} n_{i l_{d} m}=\sum_{m}\left\langle\hat{n}_{i l=l_{d} m}\right\rangle$ is the total number of interacting electrons. Since the one-electron LDA energies can be obtained from the derivatives of the total energy w.r.t. the occupation numbers of the corresponding states, the one-electron energy level for the non-interacting states of (13) is obtained as 11

$$
\varepsilon_{i l_{d} m}^{0}:=\frac{\mathrm{d}}{\mathrm{d} n_{i l_{d} m}}\left(E_{\mathrm{LDA}}-E_{\mathrm{LDA}}^{U}\right)=\varepsilon_{i l_{d} m}-U\left(n_{d}-\frac{1}{2}\right)
$$


where $\varepsilon_{i l_{d} m}$ is defined in (11) and $E_{\mathrm{LDA}}$ is the total energy calculated from $\hat{H}_{\mathrm{LDA}}$ (11).

This leads to a new Hamiltonian describing the non-interacting system

$$
H_{\mathrm{LDA}}^{0}=\sum_{i l m, j l^{\prime} m^{\prime}, \sigma}\left(\delta_{i l m, j l^{\prime} m^{\prime}} \varepsilon_{i l m}^{0} \hat{n}_{i l m}^{\sigma}+t_{i l m, j l^{\prime} m^{\prime}} \hat{c}_{i l m}^{\sigma \dagger} \hat{c}_{j l^{\prime} m^{\prime}}^{\sigma}\right),
$$

where $\varepsilon_{i l_{d} m}^{0}$ is given by (15) for the interacting orbitals and $\varepsilon_{i l m}^{0}=\varepsilon_{i l m}$ for the non-interacting orbitals. While it is not clear at present how to systematically subtract $\hat{H}_{\mathrm{LDA}}^{U}$ one should note that the subtraction of a Hartree-type energy does not substantially affect the overall behavior of a strongly correlated paramagnetic metal in the vicinity of a Mott-Hubbard metal-insulator transition (see also Sec. 2.6).

In the following it is convenient to work in reciprocal space where the matrix elements of $\hat{H}_{\mathrm{LDA}}^{0}$ are given by

$$
\left(H_{\mathrm{LDA}}^{0}(\mathbf{k})\right)_{q l m, q^{\prime} l^{\prime} m^{\prime}}=\left(H_{\mathrm{LDA}}(\mathbf{k})\right)_{q l m, q^{\prime} l^{\prime} m^{\prime}}-\delta_{q l m, q^{\prime} l^{\prime} m^{\prime}} \delta_{q l, q_{d} l_{d}} U\left(n_{d}-\frac{1}{2}\right) .(17
$$

Here, $q$ is an index of the atom in the elementary unit cell, $\left(H_{\mathrm{LDA}}(\mathbf{k})\right)_{q l m, q^{\prime} l^{\prime} m^{\prime}}$ is the matrix element of (11) in k-space, and $q_{d}$ denotes the atoms with interacting orbitals in the unit cell. The non-interacting part, $\hat{H}_{\mathrm{LDA}}^{0}$, supplemented with the local Coulomb interaction forms the (approximated) $a b$ initio Hamiltonian for a particular material under investigation:

$$
\hat{H}_{\mathrm{LDA}+\text { correl }}=H_{\mathrm{LDA}}^{0}+\sum_{i=i_{d}, l=l_{d}, m \sigma m^{\prime} \sigma}^{\prime} U_{m m^{\prime}}^{\sigma \sigma^{\prime}} \hat{n}_{i l m \sigma} \hat{n}_{i l m^{\prime} \sigma^{\prime}}
$$

To make use of this ab initio Hamiltonian it is still necessary to determine the Coulomb interaction $U$. To this end, one can calculate the LDA ground state energy for different numbers of interacting elecrons $n_{d}$ ("constrained LDA" 17 ) and employ Eq. (14) whose second derivative w.r.t. $n_{d}$ yields $U$. However, one should keep in mind that, while the total LDA spectrum is rather insensitive to the choice of the basis, the calculation of $U$ strongly depends on the shape of the orbitals which are considered to be interacting. Thus, an appropriate basis like LMTO is mandatory and, even so, some uncertainty in $U$ remains.

\subsection{Dynamical mean-field theory}

The many-body extension of LDA, Eq. (18), was proposed by Anisimov et al 11 in the context of their $\mathrm{LDA}+\mathrm{U}$ approach. Within $\mathrm{LDA}+\mathrm{U}$ the Coulomb interactions of (18) are treated within the Hartree-Fock approximation; hence it does not contain true many-body physics. While $\mathrm{LDA}+\mathrm{U}$ is successful in describing longrange ordered, insulating states of correlated electronic systems it fails to describe strongly correlated paramagnetic states. To go beyond LDA $+\mathrm{U}$ and capture the many-body nature of the electron-electron interaction, i.e., the frequency dependence of the self-energy, various approximation schemes have been proposed and 


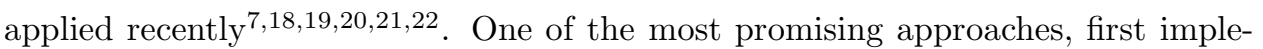

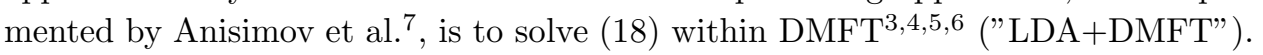
Of all extensions of LDA only the LDA+DMFT approach is presently able to describe the physics of strongly correlated, paramagnetic metals with well-developed upper and lower Hubbard bands and a narrow quasiparticle peak, which is determined by the vicinity of a Mott-Hubbard metal-insulator transition.

During the last ten years DMFT has proved to be a successful approach to investigate strongly correlated systems with local Coulomb interactions exact in the limit of high lattice coordination numbers 3 and preserves the dynamics of local interactions. Hence, it represents a dynamical mean-field approximation. In this non-perturbative approach the lattice problem is mapped onto an effective single-site problem which has to be determined self-consistently together with the k-integrated Dyson equation connecting the self energy $\Sigma$ and the Green function $G$ at frequency $\omega$ :

$$
\begin{gathered}
G_{q l m, q^{\prime} l^{\prime} m^{\prime}}(\omega)=\frac{1}{V_{B}} \int d^{3} k\left[\omega \delta_{q l m, q^{\prime} l^{\prime} m^{\prime}}-\left(H_{\mathrm{LDA}}^{0}(\mathbf{k})\right)_{q l m, q^{\prime} l^{\prime} m^{\prime}}\right. \\
\left.-\delta_{q l, q_{d} l_{d}} \Sigma_{q l m, q^{\prime} l^{\prime} m^{\prime}}(\omega)\right]^{-1} .
\end{gathered}
$$

Here, $[\ldots]^{-1}$ implies the inversion of the matrix with elements $n(=q l m), n^{\prime}\left(=q^{\prime} l^{\prime} m^{\prime}\right)$, and integration extends over the Brillouin zone with volume $V_{B}$.

The DMFT single-site problem depends on $\mathcal{G}^{-1}=G^{-1}+\Sigma$ and is equivalent to an Anderson impurity problem2324 if its hybridization $\Delta(\omega)$ satisfies $\mathcal{G}^{-1}(\omega)=$ $\omega-\int d \omega^{\prime} \Delta\left(\omega^{\prime}\right) /\left(\omega-\omega^{\prime}\right)$. The local one-particle Green function at a Matsubara frequency $\omega_{\nu}=(2 \nu+1) \pi / \beta$, orbital index $m\left(l=l_{d}, q=q_{d}\right)$, and spin $\sigma$ is given by the following functional integral over Grassmann variables $\psi$ and $\psi^{*}$ :

$$
G_{\nu m}^{\sigma}=-\frac{1}{\mathcal{Z}} \int \mathcal{D}[\psi] \mathcal{D}\left[\psi^{*}\right] \psi_{\nu m}^{\sigma} \psi_{\nu m}^{\sigma *} e^{\mathcal{A}\left[\psi, \psi^{*}, \mathcal{G}^{-1}\right]}
$$

Here, the single-site action $\mathcal{A}$ has the form

$$
\begin{aligned}
\mathcal{A}\left[\psi, \psi^{*}, \mathcal{G}^{-1}\right]=\sum_{\nu, \sigma, m} \psi_{\nu m}^{\sigma *}\left(\mathcal{G}_{\nu m}^{\sigma}\right)^{-1} \psi_{\nu m}^{\sigma} \\
-\frac{1}{2} \sum_{m \sigma, m \sigma^{\prime}}^{\prime} U_{m m^{\prime}}^{\sigma \sigma^{\prime}} \int_{0}^{\beta} d \tau \psi_{m}^{\sigma *}(\tau) \psi_{m}^{\sigma}(\tau) \psi_{m^{\prime}}^{\sigma^{\prime} *}(\tau) \psi_{m^{\prime}}^{\sigma^{\prime}}(\tau) .
\end{aligned}
$$

Due to its equivalence to an Anderson impurity problem a variety of approximative techniques have been emploved to solve the DMFT equations, such as the iterated perturbation theory (IPT)23, 3 and the non-crossing approximation (NCA) 25.26.27, as well as numerical techniques like quantum Monte-Carlo simulations (QMC) exact diagonalization (ED) 29,6, or numerical renormalization group (NRG) 30. IPT is non-self-consistent second-order perturbation theory in $U$ for the Anderson impurity problem (20) at half-filling. It represents an ansatz that also yields the correct perturbational $U^{2}$-term and the correct atomic limit for the self-energy off halffilling 31. NCA is a resolvent perturbation theory in the hybridization parameter 
$\Delta(\omega)$ of the Anderson impurity problem. Thus, it is reliable if the Coulomb interaction $U$ is large compared to the band-width. In essence, the QMC technique maps the interacting electron problem (20) onto a sum of non-interacting problems by means of Hubbard-Stratonovich transformations and evaluates this sum by Monte-Carlo sampling 22 . ED directly diagonalizes the Anderson impurity problem at a limited number of lattice sites. NRG first replaces the conduction band by a discrete set of states at $D \Lambda^{-n}(D$ : bandwidth; $n=0, \ldots, \mathcal{N})$ and then diagonalizes this problem iteratively with increasing accuracy at low energies, i.e., with increasing $\mathcal{N}$.

In principle, QMC, ED, and NRG are exact methods, but they require an extrapolation, i.e., the discretization of the imaginary time $\Delta \tau \rightarrow 0$ (QMC), the number of lattice sites of the respective impurity model $n_{s} \rightarrow \infty$ (ED), or the parameter for logarithmic discretization of the conducting band $\Lambda \rightarrow 1$ (NRG), respectively.

In the present paper, we will not present further details of these methods and

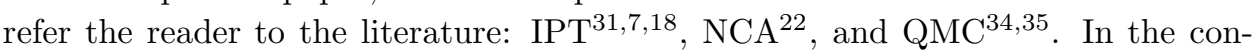
text of LDA+DMFT we refer to the computational schemes to solve the DMFT equations discussed above as $\mathrm{LDA}+\operatorname{DMFT}(\mathrm{X})$ where $\mathrm{X}=\mathrm{IPT}$, $\mathrm{NCA}_{22}$, QMd3 have been investigated in the case of $\mathrm{La}_{1} \mathrm{Sr}_{x} \mathrm{TiO}_{3}$. The same strategy was formulated by Lichtenstein and Katsnelson 18 as one of their LDA++ approaches. Lichtenstein and Katsnelson applied LDA+DMFT(IPT) 36, and were the first to use LDA+DMFT(QMC)37, to investigate the spectral properties of iron. Liebsch and Lichtenstein also applied LDA+DMFT(QMC) to calculate the photoemission spectrum of $\mathrm{Sr}_{2} \mathrm{RuO}_{4} 38$.

\subsection{Self-consistent $L D A+D M F T$}

In general, the DMFT solution will result in a change of the occupation of the different bands involved. This changes the electron density $\rho(\mathbf{r})$ and, thus, results in a new LDA-Hamiltonian $H_{\mathrm{LDA}}$ (11) since $H_{\mathrm{LDA}}$ depends on $\rho(\mathbf{r})$. At the same time also the Coulomb interaction $U$ changes and needs to be determined by a new constrained LDA calculation. In a self-consistent LDA+DMFT scheme, $H_{\mathrm{LDA}}$ and $U$ define a new Hamiltonian (18) which again needs to be solved within DMFT, etc., until convergence is reached:

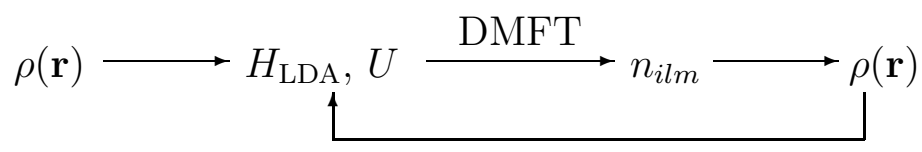

Without Coulomb interaction $(U=0)$ this scheme reduces to the self-consistent solution of the Kohn-Sham equations.

\subsection{Simplifications for transition-metal oxides}

Many transition metal oxides are cubic perovskites, with only a slight distortion of the cubic crystal structure. In these systems the transition metal $d$-orbitals lead to strong Coulomb interactions between the electrons. The cubic crystal-field of the 
oxygen causes the $d$-orbitals to split into three degenerate $t_{2 g^{-}}$and two degenerate $e_{g}$-orbitals. This splitting is often so strong that the $t_{2 g^{-}}$or $e_{g}$-bands at the Fermi energy are rather well separated from all other bands. In this situation the lowenergy physics is well described by taking only the degenerate bands at the Fermi energy into account. Without symmetry breaking, the Green function and the selfenergy of these bands remain degenerate, i.e., $G_{q l m, q^{\prime} l^{\prime} m^{\prime}}(z)=G(z) \delta_{q l m, q^{\prime} l^{\prime} m^{\prime}}$ and $\Sigma_{q l m, q^{\prime} l^{\prime} m^{\prime}}(z)=\Sigma(z) \delta_{q l m, q^{\prime} l^{\prime} m^{\prime}}$ for $l=l_{d}$ and $q=q_{d}$ (where $l_{d}$ and $q_{d}$ denote the electrons in the interacting band at the Fermi energy). Downfolding to a basis with these degenerate $q_{d}-l_{d}$-bands results in an effective Hamiltonian $H_{\mathrm{LDA}}^{0 \text { eff }}$ (where indices $l=l_{d}$ and $q=q_{d}$ are suppressed)

$$
G_{m m^{\prime}}(\omega)=\frac{1}{V_{B}} \int \mathrm{d}^{3} k\left[(\omega-\Sigma(\omega)) \delta_{m, m^{\prime}}-\left(H_{\mathrm{LDA}}^{0 \text { eff }}(\mathbf{k})\right)_{m, m^{\prime}}\right]^{-1} .
$$

Due to the diagonal structure of the self-energy the degenerate interacting Green function can be expressed via the non-interacting Green function $G^{0}(\omega)$ :

$$
G(\omega)=G^{0}(\omega-\Sigma(\omega))=\int d \epsilon \frac{N^{0}(\epsilon)}{\omega-\Sigma(\omega)-\epsilon} .
$$

Thus, it is possible to use the Hilbert transformation of the unperturbed LDAcalculated density of states (DOS) $N^{0}(\epsilon)$, i.e., Eq. (24), instead of Eq. (19). This simplifies the calculations considerably. With Eq. (24) also some conceptional simplifications arise: (i) the substraction of $\hat{H}_{\mathrm{LDA}}^{U}$ in (24) only results in an (unimportant) shift of the chemical potential and, thus, the exact form of $\hat{H}_{\mathrm{LDA}}^{U}$ is irrelevant; (ii) Luttinger's theorem of Fermi pinning holds, i.e., the interacting DOS at the Fermi energy is fixed at the value of the non-interacting DOS at $T=0$ within a Fermi liquid; (iii) as the number of electrons within the different bands is fixed, the LDA+DMFT approach is automatically self-consistent.

In this context it should be noted that the approximation Eq. (24) is justified only if the overlap between the $t_{2 g}$ orbitals and the other orbitals is rather weak.

\section{An example: $\mathrm{La}_{1-x} \mathrm{Sr}_{x} \mathrm{TiO}_{3}$}

The stoichiometric compound $\mathrm{LaTiO}_{3}$ is a cubic perovskite with a small orthorhombic distortion $\left(\angle T i-O-T i \approx 155^{\circ}\right) 39$ and is an antiferromagnetic insulator 40 below $T_{N}=125 \mathrm{~K} \pm 1$. Above $T_{N}$, or at low Sr-doping $x$, and neglecting the small orthorhombic distortion (i.e. considering a cubic structure with the same volume), $\mathrm{LaTiO}_{3}$ is a strongly correlated, but otherwise simple paramagnet with only one $3 d$-electron on the trivalent Ti sites. This makes the system a perfect trial candidate for the LDA+DMFT approach.

The LDA band-structure calculation for undoped (cubic) $\mathrm{LaTiO}_{3}$ yields the DOS shown in Fig. 11 which is typical for early transition metals. The oxygen bands, ranging from $-8.2 \mathrm{eV}$ to $-4.0 \mathrm{eV}$, are filled such that $\mathrm{Ti}$ is three-valent. Due to the crystal-field splitting, the Ti $3 d$-bands separates into two empty $e_{g}$-bands and three degenerate $t_{2 g}$-bands. Since the $t_{2 g}$-bands at the Fermi energy are well separated 


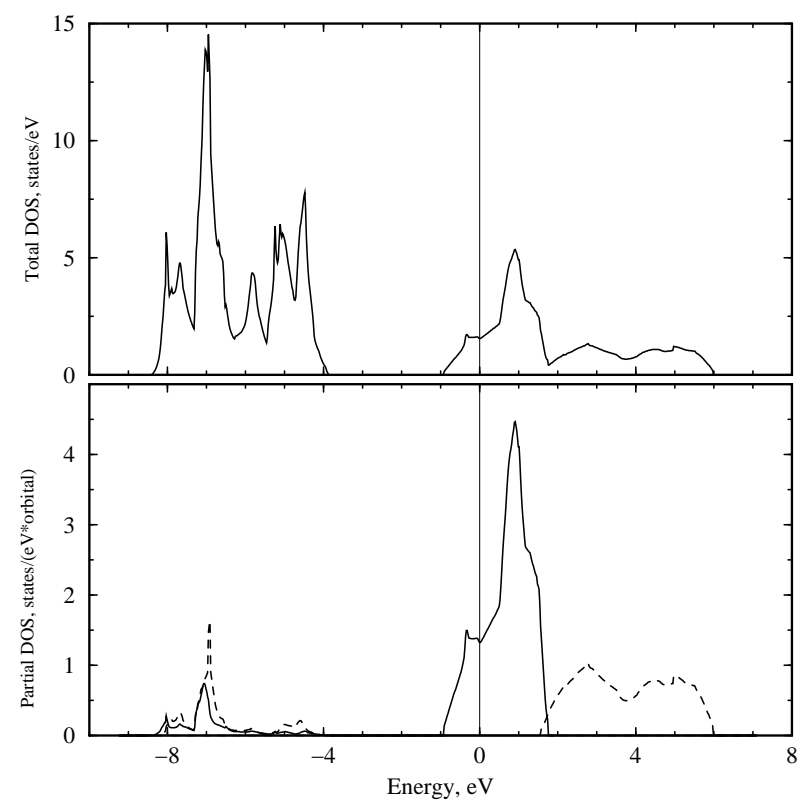

Figure 1: Densities of states of $\mathrm{LaTiO}_{3}$ calculated with LDA-LMTO. Upper figure: total DOS; lower figure: partial $t_{2 g}$ (solid lines) and $e_{g}$ (dashed lines) DOS [reproduced from Ref 35$]$.

also from the other bands we employ the approximation introduced in section 2.5 which allows us to work with the LDA DOS [Eq. (24)] instead of the full one-particle Hamiltonian [Eq. (19)]. In the LDA+DMFT calculation, Sr-doping $x$ is taken into account by adjusting the chemical potential to yield $n=1-x=0.94$ electrons within the $t_{2 g}$-bands. There is some uncertainty in the LDA-calculated Coulomb interaction parameter $U \sim 4-5 \mathrm{eV}$ (for a discussion see Ref 35 ) which is here assumed to be spin- and orbital-independent. In Fig. 2, results for the spectrum of $\mathrm{La}_{0.94} \mathrm{Sr}_{0.06} \mathrm{TiO}_{3}$ as calculated by LDA+DMFT(IPT, NCA, QMC) for the same LDA DOS at $T \approx 1000 \mathrm{~K}$ and $U=4 \mathrm{eV}$ are compared 35 . In Ref 35 the formerly presented IPT and $\mathrm{NCA}_{2} 2$ spectra were recalculated to allow for a comparison at exactly the same parameters. All three methods yield the typical features of strongly correlated metallic paramagnets: a lower Hubbard band, a quasi-particle peak (note that IPT produces a quasi-particle peak only below about $250 \mathrm{~K}$ which is therefore not seen here), and an upper Hubbard band. By contrast, within LDA the correlation-induced Hubbard bands are missing and only a broad central quasiparticle band (actually a one-particle peak) is obtained (Fig. 1).

While the results of the three evaluation techniques of the DMFT equations (the approximations IPT, NCA and the numerically exact method QMC) agree on a qualitative level, Fig. 2 reveals considerable quantitative differences. In particular, the IPT quasi-particle peak found at low temperatures (see right inset of 


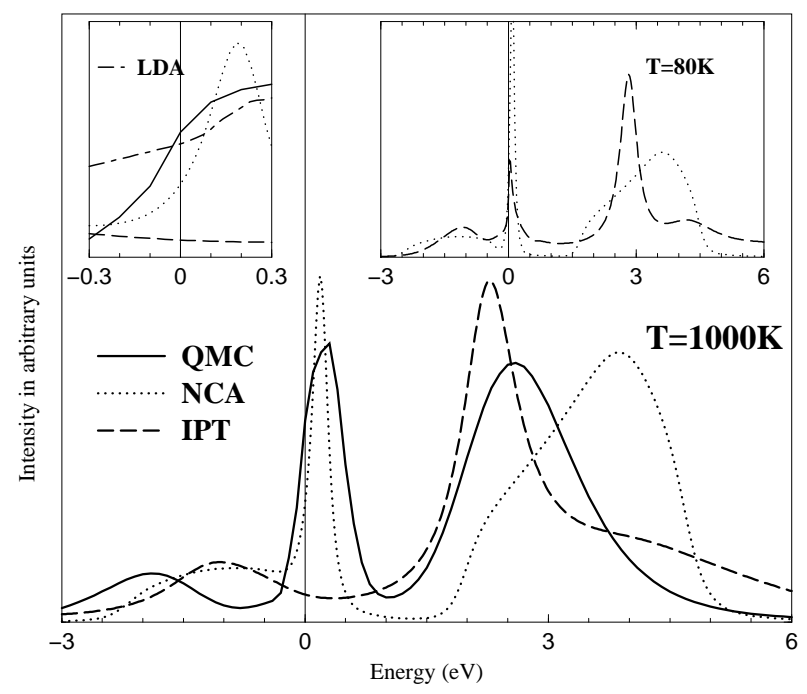

Figure 2: Spectrum of $\mathrm{La}_{0.94} \mathrm{Sr}_{0.06} \mathrm{TiO}_{3}$ as calculated by $\mathrm{LDA}+\mathrm{DMFT}(\mathrm{X})$ at $T=$ $0.1 \mathrm{eV}(\approx 1000 \mathrm{~K})$ and $U=4 \mathrm{eV}$ employing the approximations $\mathrm{X}=\mathrm{IPT}, \mathrm{NCA}$, and numerically exact QMC. Inset left: Behavior at the Fermi level including the LDA DOS. Inset right: $\mathrm{X}=\mathrm{IPT}$ and $\mathrm{NCA}$ spectra at $T=80 \mathrm{~K}$ [reproduced from Ref. 35 ].

Fig. 22 is too narrow such that it disappears already at about $250 \mathrm{~K}$ and is, thus, not present at $T \approx 1000 \mathrm{~K}$. A similarly narrow IPT quasi-particle peak was found in a three-band model study with Bethe-DOS by Kajueter and Kotlian 31. Besides underestimating the Kondo temperature, IPT also produces notable deviations in the shape of the upper Hubbard band. Although NCA comes off much better than IPT it still underestimates the width of the quasiparticle peak by a factor of two. Furthermore, the position of the quasi-particle peak is too close to the lower Hubbard band. In the left inset of Fig. 2, the spectra at the Fermi level are shown. At the Fermi level, where at sufficiently low temperatures the interacting DOS should be pinned at the non-interacting value, the NCA yields a spectral function which is almost by a factor two too small. The shortcomings of the NCA-results appear to result from the well-known problems which this approximation scheme encounters already in the single-impurity Anderson model at low temperatures and/or low frequencies 434 . Similarly, the deficiencies of the IPT-results are not entirely surprising in view of the semi-phenomenological nature of this approximation, especially for a system off half filling. This comparison shows that the choice of the method used to solve the DMFT equations is indeed important, and that, at least for the present system, the approximations IPT and NCA differ quantitatively from the numerically exact QMC.

Photoemission spectra provide a direct experimental tool to study the electronic structure and spectral properties of electronically correlated materials. A comparison of LDA+DMFT(QMC) at $1000 \mathrm{~K} 47$ with the experimental photoemis- 


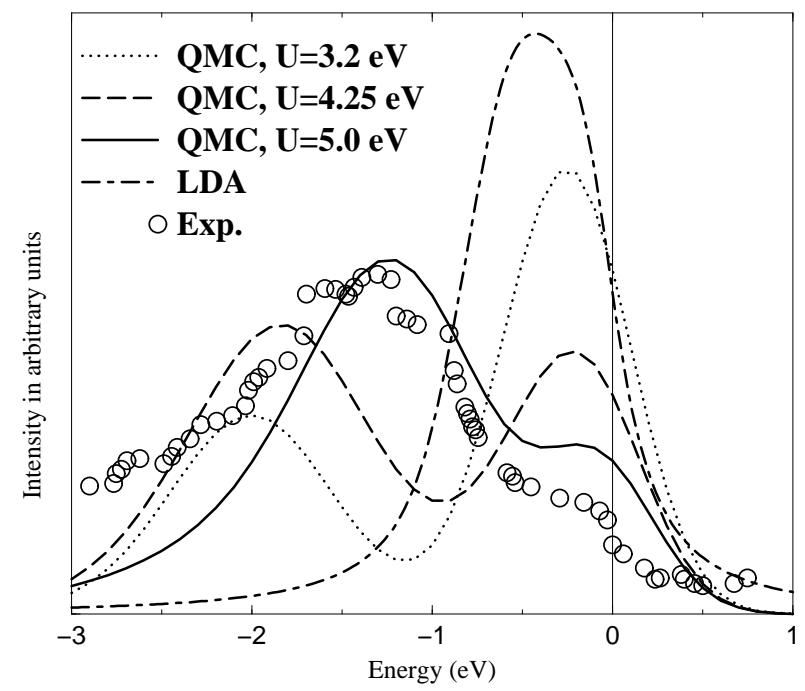

Figure 3: Comparison of the experimental photoemission spectrum 4 , the LDA result, and the LDA+DMFT(QMC) calculation for $\mathrm{La}_{0.94} \mathrm{Sr}_{0.06} \mathrm{TiO}_{3}$ (i.e., $6 \%$ hole doping) and different Coulomb interaction $U=3.2,4.25$, and $5 \mathrm{eV}$ [reproduced from Ref. .5$]$.

sion spectrum 46 of $\mathrm{La}_{0.94} \mathrm{Sr}_{0.06} \mathrm{TiO}_{3}$ is presented in Fig 3 . To take into account the uncertainty in $U 35$, we present results for $U=3.2,4.25$ and $5 \mathrm{eV}$. All spectra are multiplied with the Fermi step function and are Gauss-broadened with a broadening parameter of $0.3 \mathrm{eV}$ to simulate the experimental resolution 16 . LDA band structure calculations, the results of which are also presented in Fig. 3, clearly fail to reproduce the broad band observed in the experiment at 1-2 eV below the Fermi energy 4 . Taking the correlations between the electrons into account, this lower band is easily identified as the lower Hubbard band whose spectral weight originates from the quasi-particle band at the Fermi energy and which increases with $U$. The best agreement with experiment concerning the relative intensities of the Hubbard band and the quasi-particle peak and, also, the position of the Hubbard band is found for $U=5 \mathrm{eV}$. The value $U=5 \mathrm{eV}$ is still compatible with the $a b i n i$ tio calculation of this parameter within LDAB5. One should also bear in mind that photoemission experiments are sensitive to surface properties. Due to the reduced coordination number at the surface the bandwidth is likely to be smaller, and the Coulomb interaction less screened, i.e., larger. Both effects make the system more correlated and, thus, might also explain why better agreement is found for $U=5$ $\mathrm{eV}$. Besides that, also the polycrystalline nature of the sample, as well as spin and orbital 18 fluctuation not taken into account in the LDA+DMFT approach, will lead to a further reduction of the quasi-particle weight. 


\section{Conclusion}

In this paper we described the set-up and presented results of the computational scheme LDA+DMFT which merges two non-perturbative, complementary investigation techniques for many-particle systems in condensed matter physics. This approach allows one to perform $a b$ initio calculations of strongly correlated electronic systems. Using the band structure results calculated within local density approximation (LDA) as input, the missing electronic correlations are introduced by dynamical mean-field theory (DMFT). On a technical level this requires the solution of an effective self-consistent, multi-band Anderson impurity problem by some numerical method (e.g. IPT, NCA, QMC). Comparison of the photoemission spectrum of $\mathrm{La}_{1-x} \mathrm{Sr}_{x} \mathrm{TiO}_{3}$ calculated by LDA+DMFT using IPT, NCA and QMC reveal that the choice of the evaluation method is of considerable importance. Indeed, only with the numerically exact QMC quantitatively reliable results are obtained. The results of the $\mathrm{LDA}+\mathrm{DMFT}(\mathrm{QMC})$ approach are found to be in very good agreement with the experimental photoemission spectrum of $\mathrm{La}_{0.94} \mathrm{Sr}_{0.06} \mathrm{TiO}_{3}$.

The LDA+DMFT(QMC) scheme will provide a powerful tool for all future $a b$ initio investigations of real materials with strong electronic correlations. In particular, LDA+DMFT is the only existing $a b$ initio approach which is able to investigate correlated electronic systems close to a Mott transition, as well as heavy fermion and $f$-electron materials. The physical properties of such systems are characterized by the correlation-induced generation of small, Kondo-like energy scales which require the application of genuine many-body techniques.

With LDA+DMFT the band structure and model Hamiltonian communities which essentially lived separate lives so far are finally able to join forces. In the future both communities will need each other's input. Indeed, without DMFT the LDA will be limited to the investigation of weakly correlated systems, and without the LDA-calculated band structure the many-body approach will be restricted to the study of more or less oversimplified models.

\section{Acknowledgment}

We are grateful to R. Claessen, V. Eyert, J. Lægsgaard, A. Lichtenstein, A.K. McMahan, Th. Pruschke, G.A. Sawatzky, J. Schmalian, and M. Zölfl for useful discussions. This work was supported in part by the Sonderforschungsbereich 484 of the Deutsche Forschungsgemeinschaft, the Russian Foundation for Basic Research (RFFI-98-02-17275), and a Feodor-Lynen grant of the AvH foundation.

\section{References}

1. M. Born und R. Oppenheimer, 1927, Ann. Phys. (Leibzig) 84, 457.

2. R. O. Jones and O. Gunnarsson, Rev. Mod. Phys. 61, 689 (1989).

3. W. Metzner and D. Vollhardt, Phys. Rev. Lett. 62, 324 (1989).

4. D. Vollhardt in Correlated Electron Systems, edited by V. J. Emery, World Scientific, Singapore, 1993, p. 57. 
5. Th. Pruschke, M. Jarrell, and J. K. Freericks, Adv. in Phys. 44, 187 (1995).

6. A. Georges, G. Kotliar, W. Krauth, and M. J. Rozenberg, Rev. Mod. Phys. 68, 13 (1996).

7. V. I. Anisimov, A. I. Poteryaev, M. A. Korotin, A. O. Anokhin, and G. Kotliar, J. Phys. Cond. Matter 9, 7359 (1997).

8. P. Hohenberg and W. Kohn, Phys. Rev. B 136, 864 (1964)

9. M. Levy, Proc. Natl. Acad. Sci. (USA), 76, 6062 (1979).

10. W. Kohn and L. J. Sham, Phys. Rev. 140, 4A, A1133 (1965); W. Kohn, L.J. Sham, Phys. Rev. A - Gen.Phys. 140, 1133 (1965); L.J. Sham, W. Kohn, Phys. Rev. 145 N 2, 561 (1966).

11. V. I. Anisimov, J. Zaanen, and O. K. Andersen, Phys. Rev. B 44, 943 (1991); V. I. Anisimov, F. Aryasetiawan, and A. I. Lichtenstein, J. Phys. Cond. Matter 9, 767 (1997).

12. O. K. Andersen, Phys. Rev. B 12, 3060 (1975); O. Gunnarsson, O. Jepsen, and O. K. Andersen, Phys. Rev. B 27, 7144 (1983).

13. T. C. Leung, X. W. Wang, and B. N. Harmon, Phys. Rev. B 37, 384 (1988); W. E. Pickett, Rev. Mod. Phys. 61, 433 (1989).

14. Note, that the largest non-local contribution is the nearest-neighbor density-density interaction which, to ding order in $Z$ ( $Z$ : number of nearest-neighbor sites), yields only the Hartree term 16 which is already taken into account in the LDA.

15. L. Hedin and B. Lundqvist J. Phys. C: Solid State Phys. 4, 2064 (1971); U. von Barth and L. Hedin J. Phys. C: Solid State Phys. 5, 1629 (1972).

16. E. Müller-Hartmann, Z. Phys. B 74, 507 (1989); J. Wahle et al. Phys. Rev. B 58, 12749 (1998).

17. O. Gunnarson, O. K. Andersen, O. Jepsen, and J. Zaanen, Phys. Rev. B 39, 1708 (1989).

18. A. I. Lichtenstein and M. I. Katsnelson, Phys. Rev. B 57, 6884 (1998).

19. V. Drchal, V. Janiš, and J. Kudrnovský in Electron Correlations and Material Properties, edited by A. Gonis, N. Kioussis, and M. Ciftan, Kluwer/Plenum, New York 1999, p. 273.

20. J. Lægsgaard and A. Svane, Phys. Rev. B 58, 12817 (1998).

21. Th. Wolenski, Combining bandstructure and dynamical mean-field theory: A new perspective on $V_{2} O_{3}$, PhD Thesis, Universität Hamburg 1998, Shaker Verlag, Aachen 1999.

22. M. B. Zölf, Th. Pruschke, J. Keller, A. I. Poteryaev, I. A. Nekrasov, and V. I. Anisimov, Phys. Rev. B 61, 12810 (2000).

23. A. Georges and G. Kotliar, Phys. Rev. B 45, 6479 (1992).

24. M. Jarrell, Phys. Rev. Lett. 69, 168 (1992).

25. H. Keiter and J. C. Kimball, Phys. Rev. Lett. 25, 672 (1970); N. E. Bickers, D. L. Cox, and J. W. Wilkins, Phys. Rev. B 36, 2036 (1987).

26. Th. Pruschke and N. Grewe, Z. Phys. B 74, 439 (1989).

27. Th. Pruschke, D. L. Cox, and M. Jarrell, Phys. Rev. B 47, 3553 (1993).

28. J. E. Hirsch and R. M. Fye, Phys. Rev. Lett. 56, 2521 (1986); M. Jarrell, Phys. Rev. Lett. 69, 168 (1992); M. Rozenberg, X. Y. Zhang, and G. Kotliar, Phys. Rev. Lett. 69, 1236 (1992); A. Georges and W. Krauth, Phys. Rev. Lett. 69, 1240 (1992).

29. M. Caffarel and W. Krauth, Phys. Rev. Lett. 72, 1545 (1994).

30. R. Bulla, to appear in Adv. Sol. State Phys. (2000), preprint cond-mat/0003377.

31. H. Kajueter and G. Kotliar, Int. J. Mod. Phys. 11, 729 (1997).

32. We note that QMC is restricted to temperatures of the order of $T=0.1 \mathrm{eV}(\approx 10 \rho \mathrm{K})$ on current workstations. Furthermore, it relies on the maximum entropy method $B 3$ for the analytical continuation from Matsubara to real frequencies. This method is reliable in the vicinity of the Fermi energy whereas its energy resolution becomes poorer at 
larger energies.

33. M. Jarrell and J. E. Gubernatis, Physics Reports 269, 133 (1996).

34. M. J. Rozenberg, Phys. Rev. B 55, R4855 (1997); J. E. Han, M. Jarrell, and D. L. Cox, Phys. Rev. B 58, R4199 (1998); K. Held and D. Vollhardt, Euro. Phys. J. B 5, 473 (1998).

35. I. A. Nekrasov, K. Held, N. Blümer, A. I. Poteryaev, V. I. Anisimov, and D. Vollhardt, preprint cond-mat/0005207, Euro Phys. J B in print.

36. M. I. Katsnelson and A. I. Lichtenstein, J. Phys. Cond. Matter 11, 1037 (1999).

37. M. I. Katsnelson and A. I. Lichtenstein, preprint cond-mat/9904428 (1999).

38. A. Liebsch and A. Lichtenstein, Phys. Rev. Lett. 84, 1591 (2000).

39. D. A. MacLean, H.-N. Ng, and J. E. Greedan, J. Solid State Chem. 30, 35 (1979).

40. M. Eitel and J. E. Greedan, Journal of the Less-Common Metals 116, 95 (1986).

41. J. P. Gopel, J. E. Greedan, and D. A. MacLean, J. Solid State Chem. 43, 244 (1981).

42. Y. Okimoto, T. Katsufuji, Y. Okada, T. Arima, and Y. Tokura, Phys. Rev. B 51, 9581 (1995).

43. E. Müller-Hartmann, Z. Phys. B 57, 281 (1984).

44. NCA is a self-consistent perturbation theory for the single-impurity Anderson model and is often a fast method to obtain results for this model and also within DMFT. However, NCA is known to violate Fermi liquid properties at temperatures much lower than the smallest energy scale of the problem and whenever charge excitations become important 43.45 . Hence, in some parameter ranges it fails to account for the proper low-energy physics and must therefore be applied with considerable card 45 .

45. Th. Pruschke, D. L. Cox, and M. Jarrell, Phys. Rev. B 47, 3553 (1993).

46. A. Fujimori et al, Phys. Rev. Lett. 69, 1796 (1992). A. Fujimori et al, Phys. Rev. B 46, 9841 (1992). A qualitatively and quantitatively similar spectrum was obtained recently by Yosida et al., preprint cond-mat/9911446, with an experimental energy resolution of only $30 \mathrm{meV}$. One may suppose, however, that broadening effects due to the polycrystalline nature of the sample and surface effects are, then, larger than the instrumental resolution.

47. At present, QMC simulations of the DMFT equations are not feasible at the experimental temperature $(80 \mathrm{~K})$. We note however, that no intrinsic temperature dependence was observed in the experiment 16 , at least up to room temperature.

48. B. Keimer et al., preprint cond-mat/0002014.

49. J.P. Perdew and A. Zunger, Phys. Rev. B 23, 5048 (1981). A. Svane and O. Gunnarsson, Phys. Rev. Lett 65, 1148 (1990).

50. F. Aryasetiawan and O. Gunnarsson, Rep. Prog. Phys. 61, 237-312 (1998). 